\title{
ADDITION FORMULAS FOR $q$-LEGENDRE-TYPE FUNCTIONS
}

\author{
Mizan Rahman and Qazi M. Tariq
}

Dedicated to Richard Askey on the occasion of his 65th birthday

\begin{abstract}
Two families of addition formula for $q$-Legendre-type polynomials are obtained by application of known product formulas of Askey-Wilson and $q$-Racah polynomials. It is shown that the formula involving the $q$-Racah polynomials also leads to a $q$-analogue of the classical addition formula for the nonterminating Legendre functions. Unlike the quantum group-theoretic methods used by Koelink to obtain essentially the same formulas, our method is purely analytic based on $q$-series techniques.
\end{abstract}

\section{Introduction}

The purpose of this paper is to show how to obtain, by purely analytical methods, families of addition formulas which, in appropriate limits, approach Laplace's [14] addition formula for Legendre polynomials

$$
\begin{aligned}
& P_{n}(\cos \theta \cos \varphi+\sin \theta \sin \varphi \cos \psi) \\
& \quad=P_{n}(\cos \theta) P_{n}(\cos \varphi)+2 \sum_{m=1}^{n} \frac{(n-m) !}{(n+m) !} P_{n}^{m}(\cos \theta) P_{n}^{m}(\cos \varphi) \cos m \psi
\end{aligned}
$$

where $P_{n}(x)$ is the Legendre polynomial of degree $n$ in $x,|x|<1$, and

$$
P_{n}^{m}(x)=(-1)^{m}\left(1-x^{2}\right)^{\frac{m}{2}} \frac{d^{m}}{d x^{m}} P_{n}(x)
$$

is the associated Legendre polynomials; see [21]. Gegenbauer [9] found an extension of (1.1) for the ultraspherical (also called Gegenbauer) polynomials $C_{n}^{\lambda}(x)$ :

$$
\begin{aligned}
& C_{n}^{\lambda}(\cos \theta \cos \varphi+\sin \theta \sin \varphi \cos \psi)=\frac{n !}{(2 \lambda)_{n}} C_{n}^{\lambda}(\cos \theta) C_{n}^{\lambda}(\cos \varphi) \\
& \quad+\sum_{k=1}^{n} \frac{2 k+2 \lambda-1}{2 \lambda-1} \frac{(n-k) !(\lambda)_{k}^{2} 2^{2 k}}{(2 \lambda)_{n+k}} C_{n-k}^{\lambda+k}(\cos \theta) C_{n-k}^{\lambda+k}(\cos \varphi) C_{k}^{\lambda-\frac{1}{2}}(\cos \psi)
\end{aligned}
$$

(see [21]), which reduces to (1.1) as $\lambda \rightarrow \frac{1}{2}$ since

$$
P_{n}(x)=C_{n}^{\frac{1}{2}}(x) \text { and } \lim _{\lambda \rightarrow 0} \frac{k+\lambda}{\lambda} C_{k}^{\lambda}(\cos \psi)= \begin{cases}2 \cos k \psi, & k=1,2, \ldots, \\ 1, & k=0 .\end{cases}
$$

Received February 20, 1998.

1991 Mathematics Subject Classification: Primary 33D45, Secondary 33D80.

Key words and phrases: $q$-Legendre polyomials and functions, $q$-Racah and Askey-Wilson polynomials, little and big $q$-Jacobi polynomials, balanced and very-well-poised basic hypergeometric series, Askey-Wilson integral, product and addition formulas. 
There have been many published proofs of (1.3) since its first appearance in 1875, some analytic and some algebraic. Algebraic proofs often rely on group-theoretic techniques since addition formulas of special functions usually have group-theoretic interpretations. For example, Vilenkin's proof [19] is based on the irreducible unitary representations of the group $S U(2)$. Generally, an addition formula is a statement of the homomorphism property

$$
t_{j, k}\left(g_{1} g_{2}\right)=\sum_{l} t_{j, l}\left(g_{1}\right) t_{l, k}\left(g_{2}\right)
$$

where $g_{1}, g_{2} \in G$, and $t_{j, k}(g)=\left\langle T(g) e_{j}, e_{k}\right\rangle$ are the matrix elements of the representation $T$ of the group $G$ on a Hilbert space $V$ with respect to a suitably chosen basis $\left\{e_{n}\right\}$; see Vilenkin [19], Vilenkin and Klimyk [20], and Koelink [10]. For further references on (1.3), see Askey [1]. Among many applications of (1.3) is Gegenbauer's product formula [9]

$$
\frac{C_{n}^{\lambda}(x) C_{n}^{\lambda}(y)}{C_{n}^{\lambda}(1) C_{n}^{\lambda}(1)}=\int_{-1}^{1} K(x, y, z) \frac{C_{n}^{\lambda}(z)}{C_{n}^{\lambda}(1)} d z
$$

where

$$
K(x, y, z)=\frac{\Gamma\left(\lambda+\frac{1}{2}\right)}{\Gamma\left(\frac{1}{2}\right) \Gamma(\lambda)} \frac{\left(1-x^{2}-y^{2}-z^{2}+2 x y z\right)^{\lambda-1}}{\left[\left(1-x^{2}\right)\left(1-y^{2}\right)\right]^{\lambda-\frac{1}{2}}} \quad \text { or } \quad 0
$$

depending on whether $1-x^{2}-y^{2}-z^{2}+2 x y z$ is positive or negative, respectively. This and an extension of it to the Jacobi polynomials due to Koornwinder as well as Gasper (see Askey [1]) were the basis of Gasper's elaborate study [6, 7] of Banach algebras for Jacobi series. Product formulas of the type (1.6) lead to integral representations of orthogonal polynomials which, in turn, can be used to compute, for example, generating functions of these polynomials.

With the advent of quantum groups and quantum algebras in the second half of the 1980s, the quest for addition formulas for $q$-special functions, in general, and for the $q$-orthogonal polynomials, in particular, has naturally taken a different turn. For a brief review of the different approaches to the same question, see Koelink [10] and the references therein. Before the start of the quantum era, addition formulas for $q$-Krawtchouk and $q$-Hahn polynomials were found by Dunkl [4] and Stanton [17], through an interpretation of them on Lie-type finite groups. Rahman and Verma [15] also found $q$-versions of (1.3) and (1.6) by purely analytic methods. However, Koornwinder's addition formula [13] for the little $q$-Legendre polynomials:

$$
\begin{aligned}
& p_{n}\left(q^{z} ; 1,1 \mid q\right) W_{y}\left(q^{z} ; q^{x} \mid q\right)=p_{n}\left(q^{y} ; 1,1 \mid q\right) p_{n}\left(q^{x+y} ; 1,1 \mid q\right) W_{y}\left(q^{z} ; q^{x} \mid q\right) \\
& +\sum_{k=1}^{n} \frac{(q ; q)_{x+y+k}(q ; q)_{n+k} q^{k(y+k-n)}}{(q ; q)_{x+y}(q ; q)_{n-k}(q ; q)_{k}^{2}} \\
& \quad \times p_{n-k}\left(q^{y} ; q^{k}, q^{k} \mid q\right) p_{n-k}\left(q^{x+y} ; q^{k}, q^{k} \mid q\right) W_{y+k}\left(q^{z} ; q^{x} \mid q\right) \\
& +\sum_{k=1}^{n} \frac{(q ; q)_{y}(q ; q)_{n+k} q^{k(x+y+1-n)}}{(q ; q)_{y-k}(q ; q)_{n-k}(q ; q)_{k}^{2}} \\
& \quad \times p_{n-k}\left(q^{y-k} ; q^{k}, q^{k} \mid q\right) p_{n-k}\left(q^{x+y-k} ; q^{k}, q^{k} \mid q\right) W_{y-k}\left(q^{z} ; q^{x} \mid q\right)
\end{aligned}
$$

where the little $q$-Jacobi polynomials are defined by

$$
p_{n}(x ; a, b \mid q)={ }_{2} \varphi_{1}\left(q^{-n}, a b q^{n+1} ; a q ; q, q x\right),
$$


$n=0,1,2, \ldots$, and the Wall polynomials by

$$
W_{n}(x ; a \mid q)=p_{n}(x ; a, 0 \mid q)
$$

(see [8]), provides convincing evidence that the group-theoretic method is the more natural method of discovering addition theorems because it would be almost impossible to suspect a formula of the form (1.8) by analytic considerations alone. However, once an addition formula is discovered by algebraic techniques, usually it is not too difficult to find an alternative analytic proof as, for example, Rahman's proof [16] of (1.8) would testify. The converse does not occur too often, but occasionally it can. Rahman and Verma's full addition formula [15] for the continuous $q$-ultraspherical polynomials so far has not been proved directly by the quantum group or quantum algebra method although the related $q$-Legendre-type cases have been proved by quite a number of authors; see Koelink [10] for references. The fact that (1.8) is a $q$-extension of (1.1) is not at all obvious. One has to be able to retrieve (1.1) from (1.8) by taking the limit $q \rightarrow 1$ after having made appropriate substitutions. This rather delicate limiting procedure was carried out by Van Assche and Koornwinder [18].

In [12], however, Koelink mentions how to derive the Rahman-Verma formula from the $q$-Legendre case by using $q$-derivatives and analytic continuation. Koelink [11] also found an addition formula for the big $q$-Legendre polynomials:

$$
P_{n}(x ; 1,1,-c \mid q)={ }_{3} \varphi_{2}\left[\begin{array}{c}
q^{-n}, q^{n+1}, x \\
q,-c q
\end{array} ; q, q\right]
$$

(see [8]). Koelink's review article [10] contains a sort of master addition formula from which these addition formulas for $q$-Legendre-type polynomials emerge as special cases.

The main objective of this paper is to derive two such master formulas, one for the continuous and the other for the discrete case, by simple $q$-series methods, from which all the known addition formulas for $q$-Legendre-type polynomials can be obtained as special and/or limiting cases. A $q$-series or a basic hypergeometric series is a series of the type $\sum u_{r}$ where $u_{r}$ is a rational function of $q^{r}$.

Denoting the $q$-shifted factorials

$$
\begin{gathered}
(a ; q)_{n}= \begin{cases}(1-a)(1-a q) \cdots\left(1-a q^{n}\right), & n=1,2, \ldots \\
1 & n=0\end{cases} \\
\left(a_{1}, \ldots, a_{k} ; q\right)_{n}=\prod_{j=1}^{k}\left(a_{j} ; q\right)_{n},
\end{gathered}
$$

a $q$-series of $r+1$ numerator and $r$ denominator parameters is defined by

$$
\begin{aligned}
& { }_{r+1} \varphi_{r}\left(a_{1}, a_{2}, \ldots, a_{r+1} ; b_{1}, b_{2}, \ldots, b_{r} ; q, z\right) \\
& \quad \equiv{ }_{r+1} \varphi_{r}\left[\begin{array}{c}
a_{1}, a_{2}, \ldots, a_{r+1} \\
b_{1}, b_{2}, \ldots, b_{r}
\end{array} ; q, z\right]:=\sum_{k=0}^{\infty} \frac{\left(a_{1}, a_{2}, \ldots, a_{r+1} ; q\right)_{k}}{(q ; q)_{k}\left(b_{1}, b_{2}, \ldots, b_{r} ; q\right)_{k}} z^{k} .
\end{aligned}
$$

If any of the numerator parameters is of the form $q^{-n}$ where $n$ is a nonnegative integer and there is no zero factor in the denominator, then the above series becomes a polynomial of degree $n$ in $z$. In the nonpolynomial case, we need to assume that $|z|<1$ and that $|q|<1$ for the sake of convergence. We shall assume throughout this paper that $|q|<1$. We shall use some special but standard types of $q$-series, like a balanced and a very-well-poised series. The $q$-series in (1.14) is balanced if 
$z=q$ and $b_{1} b_{2} \cdots b_{r}=q a_{1} a_{2} \cdots a_{r+1}$; it is a very-well-poised series if $a_{2}=q a_{1}^{1 / 2}$, $a_{3}=-q a_{1}^{1 / 2}, b_{k}=q a_{1} / a_{k+1}, k=1,2, \ldots, r$. We also shall use a special notation for a very-well-poised series:

$$
\begin{aligned}
& { }_{r+1} W_{r}\left(a_{1} ; a_{4}, a_{5}, \ldots, a_{r+1} ; q, z\right) \\
& :={ }_{r+1} \varphi_{r}\left[\begin{array}{c}
a_{1}, q \sqrt{a_{1}},-q \sqrt{a_{1}}, a_{4}, \ldots, a_{r+1} \\
\sqrt{a_{1}},-\sqrt{a_{1}}, q a_{1} / a_{4}, \ldots, q a_{1} / a_{r+1}
\end{array} ; q, z\right] .
\end{aligned}
$$

See [8] for notations, definitions, and properties of these special $q$-series.

In Section 3, we shall derive the following addition formula in the continuous case:

$$
\begin{gathered}
p_{n}(x ; d, q / d, b, q / b) p_{m}(x ; a, b, c, d)=A_{0}(m, n, a, b, c, d) p_{m}(x ; a, b, c, d) \\
+\sum_{k=1}^{n} A_{k}(m, n, a, b, c, d) p_{m+k}(x ; a, b, c, d) \\
+\sum_{k=1}^{\min (m, n)} A_{k}^{\prime}(m, n, a, b, c, d) p_{m-k}(x ; a, b, c, d)
\end{gathered}
$$

where

$$
\begin{aligned}
& A_{0}(m, n, a, b, c, d)=\frac{\left(a b c d q^{2 m} ; q\right)_{n}}{\left(q^{2-2 m} / a b c d ; q\right)_{n}}\left(q^{1-2 m} / a b c d\right)^{n} \\
& \times{ }_{4} \varphi_{3}\left[\begin{array}{c}
q^{-n}, q^{n+1}, a d q^{m}, c d q^{m} \\
q, d q / b, a b c d q^{2 m}
\end{array} ; q, q\right] \\
& \times{ }_{4} \varphi_{3}\left[\begin{array}{c}
q^{-n}, q^{n+1}, b d q^{m}, a b c d q^{m-1} \\
q, b d, a b c d q^{2 m}
\end{array} ; q, q\right], \\
& A_{k}(m, n, a, b, c, d)=\frac{\left(a b q^{m}, a c q^{m}, a d q^{m}, a b c d q^{m-1}, q^{-n}, q^{n+1} ; q\right)_{k}}{(q, q, b d, d q / b ; q)_{k}\left(a b c d q^{2 m-1} ; q\right)_{2 k}}(-d / a)^{k} q^{\left(\begin{array}{c}
k+1 \\
2
\end{array}\right)} \\
& \times \frac{\left(a b c d q^{2 m+2 k} ; q\right)_{n-k}}{\left(q^{2-2 m} / a b c d ; q\right)_{n-k}}\left(q^{1-2 m-k} / a b c d\right)^{n-k} \\
& \times{ }_{4} \varphi_{3}\left[\begin{array}{c}
q^{k-n}, q^{n+k+1}, a d q^{m+k}, c d q^{m+k} \\
q^{k+1}, d q^{k+1} / b, a b c d q^{2 m+2 k}
\end{array}\right] \\
& \times{ }_{4} \varphi_{3}\left[\begin{array}{c}
q^{k-n}, q^{n+k+1}, b d q^{m+k}, a b c d q^{m+k-1} \\
q^{k+1}, b d q^{k}, a b c d q^{2 m+2 k}
\end{array} ; q, q\right], \\
& A_{k}^{\prime}(m, n, a, b, c, d)=\frac{\left(q^{-m}, q^{1-m} / b c, q^{1-m} / b d, q^{1-m} / c d, q^{-n}, q^{n+1} ; q\right)_{k}}{(q, q, b d, d q / b ; q)_{k}\left(q^{-2 m+1} / a b c d ; q\right)_{2 k}}(-d / a)^{k} q^{\left(\begin{array}{c}
k+1 \\
2
\end{array}\right)} \\
& \times \frac{\left(a b c d q^{2 m} ; q\right)_{n-k}}{\left(q^{2-2 m+2 k} / a b c d ; q\right)_{n-k}}\left(q^{1-2 m+k} / a b c d\right)^{n-k} \\
& \times{ }_{4} \varphi_{3}\left[\begin{array}{c}
q^{k-n}, q^{n+k+1}, a d q^{m}, c d q^{m} \\
q^{k+1}, d q^{k+1} / b, a b c d q^{2 m}
\end{array} ; q, q\right] \\
& \times{ }_{4} \varphi_{3}\left[\begin{array}{c}
q^{k-n}, q^{n+k+1}, b d q^{m}, a b c d q^{m-1} \\
q^{k+1}, b d q^{k}, a b c d q^{2 m}
\end{array} ; q, q\right],
\end{aligned}
$$


and

$$
p_{n}(x ; a, b, c, d)={ }_{4} \varphi_{3}\left[\begin{array}{c}
q^{-n}, a b c d q^{n-1}, a e^{i \theta}, a e^{-i \theta} \\
a b, a c, a d
\end{array} ; q, q\right]
$$

are the Askey-Wilson polynomials introduced in [3] with a different normalization (also see [8]) and $x=\cos \theta, 0 \leq \theta \leq \pi$. It is not difficult to see that (1.16) is actually a terminating version of Koelink's general addition formula in [10, Theorem 4.1].

The second addition formula that we shall prove in Section 5 is

$$
\begin{aligned}
& { }_{4} \varphi_{3}\left[\begin{array}{c}
q^{-\nu}, q^{\nu+1}, q^{z-N}, q^{-z} / c \\
q, q^{-N}, q / c
\end{array} q, q\right] R_{m}\left(q^{z} ; a, b, c, q^{-N}\right) \\
& =B_{0}\left(\nu, m, a, b, c, q^{-N}\right) R_{m}\left(q^{z} ; a, b, c, q^{-N}\right) \\
& \quad+\sum_{k=1}^{\infty} B_{k}\left(\nu, m, a, b, c, q^{-N}\right) R_{m+k}\left(q^{z} ; a, b, c, q^{-N}\right) \\
& \quad+\sum_{k=1}^{m} B_{k}^{\prime}\left(\nu, m, a, b, c, q^{-N}\right) R_{m-k}\left(q^{z} ; a, b, c, q^{-N}\right)
\end{aligned}
$$

where $z=0,1 \ldots, N$, and $\nu$ is a complex parameter which is a nonnegative integer $n$ only if the ${ }_{4} \varphi_{3}$ series on the left-hand side is a polynomial of degree $n$ in $q^{N-z}$. Also

$$
R_{m}\left(q^{z} ; a, b, c, q^{-N}\right)={ }_{4} \varphi_{3}\left[\begin{array}{c}
q^{-m}, a b q^{m+1}, q^{-z}, c q^{z-N} \\
a q, q^{-N}, b c q
\end{array} ; q, q\right]
$$

is the $q$-Racah polynomial introduced in [2]; see also [8], and

$$
\begin{aligned}
& B_{0}\left(\nu, m, a, b, c, q^{-N}\right)={ }_{4} \varphi_{3}\left[\begin{array}{c}
q^{-\nu}, q^{\nu+1}, q^{-m} / a, q^{-m} / b c \\
q, q^{-2 m} / a b, q / c
\end{array} ; q, q\right] \\
& \times{ }_{4} \varphi_{3}\left[\begin{array}{c}
q^{-\nu}, q^{\nu+1}, q^{m-N}, a b q^{m+1} \\
q, q^{-N}, a b q^{2 m+2}
\end{array} ; q, q\right], \\
& \frac{\left(a b q^{m+1}, a q^{m+1}, a q^{m+1}, b c q^{m+1}, q^{m-N}, q^{-\nu}, q^{\nu+1} ; q\right)_{k}}{\left(q, q, q / c, q^{-N}, a b q^{N+m+2} ; q\right)_{k}\left(a b q^{2 m+1} ; q\right)_{2 k}}(-1 / c)^{k} q^{\left(\begin{array}{c}
k+1 \\
2
\end{array}\right)} \\
& \times{ }_{4} \varphi_{3}\left[\begin{array}{c}
q^{k-\nu}, q^{k+\nu+1}, q^{-m} / a, q^{-m} / b c \\
q^{k+1}, q^{k+1} / c, q^{-2 m} / a b
\end{array} q, q\right] \\
& \times{ }_{4} \varphi_{3}\left[\begin{array}{c}
q^{k-\nu}, q^{k+\nu+1}, q^{m+k-N}, a b q^{m+k+1} \\
q^{k+1}, q^{k-N}, a b q^{2 m+2 k+2}
\end{array} ; q, q\right] \text {. }
\end{aligned}
$$


and

$$
\begin{aligned}
& B_{k}^{\prime}\left(\nu, m, a, b, c, q^{-N}\right)= \\
& \frac{\left(a q^{m-k+1}, b q^{m-k+1}, a q^{m-k+1} / c, q^{m-k+1}, q^{-\nu}, q^{\nu+1} ; q\right)_{k}}{\left(q, q, q / c, q^{-N} ; q\right)_{k}\left(a b q^{2 m-2 k+2} ; q\right)_{2 k}}\left(-q^{-N}\right)^{k} q^{\left(\begin{array}{c}
k+1 \\
2
\end{array}\right)} \\
& \times{ }_{4} \varphi_{3}\left[\begin{array}{c}
q^{k-\nu}, q^{k+\nu+1}, q^{k-m} / a, q^{k-m} / b c \\
q^{k+1}, q^{k+1} / c, q^{2 k-2 m} / a b
\end{array}\right] \\
& \times{ }_{4} \varphi_{3}\left[\begin{array}{c}
q^{k-\nu}, q^{k+\nu+1}, q^{m-N}, a b q^{m+1} \\
q^{k+1}, q^{k-N}, a b q^{2 m+2}
\end{array} ; q, q\right] .
\end{aligned}
$$

If $\nu$ is a nonnegative integer, then (1.21) is valid for arbitrary $a, b, c$. Otherwise, we need to stipulate that the ${ }_{4} \varphi_{3}$ series in (1.23)-(1.25) all terminate, for which it is sufficient to assume that $q^{k-m} / a$ or $q^{k-m} / b c$ is of the form $q^{-n}, n=0,1,2, \ldots$, for $k=0,1, \ldots, m$.

In Section 5, we shall deduce from (1.21) the following addition formula for the little $q$-Legendre functions:

$$
\begin{aligned}
& p_{\nu}\left(q^{z} ; 1,1 \mid q\right) W_{y}\left(q^{z} ; q^{x} \mid q\right)=p_{\nu}\left(q^{y} ; 1,1 \mid q\right) p_{\nu}\left(q^{x+y} ; 1,1 \mid q\right) W_{y}\left(q^{z} ; q^{x} \mid q\right) \\
& +\sum_{k=1}^{\infty} \frac{\left(q^{-\nu}, q^{\nu+1}, q^{x+y+1} ; q\right)_{k}}{(q, q ; q)_{k}}(-1)^{k} q^{y k+\left(\begin{array}{c}
k+1 \\
2
\end{array}\right)} \\
& \quad \times p_{\nu-k}\left(q^{y} ; q^{k}, q^{k} \mid q\right) p_{\nu-k}\left(q^{x+y} ; q^{k}, q^{k} \mid q\right) W_{y+k}\left(q^{z} ; q^{x} \mid q\right) \\
& +\sum_{k=1}^{y} \frac{(q ; q)_{y}\left(q^{-\nu}, q^{\nu+1} ; q\right)_{k}}{(q ; q)_{y-k}(q, q ; q)_{k}}(-1)^{k} q^{(x+y+1) k-\left(\begin{array}{c}
k \\
2
\end{array}\right)} \\
& \quad \times p_{\nu-k}\left(q^{y-k} ; q^{k}, q^{k} \mid q\right) p_{\nu-k}\left(q^{x+y-k} ; q^{k}, q^{k} \mid q\right) W_{y-k}\left(q^{z} ; q^{x} \mid q\right)
\end{aligned}
$$

where

$$
p_{\nu}\left(q^{x} ; a, b \mid q\right)={ }_{2} \varphi_{1}\left[\begin{array}{c}
q^{-\nu}, a b q^{\nu+1} \\
a q
\end{array} ; q, q^{x+1}\right]
$$

are the little $q$-Jacobi functions that become the little $q$-Legendre functions when $a=b=1$. However, the addition formula that Floris and Koelink [5] recently obtained for the little $q$-Jacobi polynomials $p_{\nu}\left(x ; q^{\nu}, 1 \mid q\right)$ does not follow from (1.21). If $\nu$ is not a nonnegative integer then it is essential for convergence of the ${ }_{2} \varphi_{1}$ series in (1.27) that $x \geq 0$. Koornwinder's addition formula (1.8) is clearly the polynomial case of (1.26). One can show that in the nonpolynomial case, (1.26) is a $q$-analogue of the addition formula for the Legendre function:

$$
\begin{aligned}
& P_{\nu}(\cos \theta \cos \varphi+\sin \theta \sin \varphi \cos \psi)=P_{\nu}(\cos \theta) P_{\nu}(\cos \varphi) \\
&+2 \sum_{m=1}^{\infty} \frac{\Gamma(\nu-m+1)}{\Gamma(\nu+m+1)} P_{\nu}^{m}(\cos \theta) P_{\nu}^{m}(\cos \varphi) \cos m \psi
\end{aligned}
$$

where

$$
\begin{gathered}
P_{\nu}(x)=F\left(-\nu, \nu+1 ; 1 ; \frac{(1-x)}{2}\right) \\
P_{\nu}^{m}(x)=(-1)^{m}\left(1-x^{2}\right)^{\frac{m}{2}} \frac{d^{m}}{d x^{m}} P_{\nu}(x)
\end{gathered}
$$


see $[21,15.71]$. The limiting process from (1.26) to (1.28) is essentially the same as the one from (1.8) to (1.1) carried out in [18].

The proof of (1.16) depends on the evaluation of an integral over products of AskeyWilson polynomials which we shall carry out in Section 2. In Section 4, we shall compute sums over products of $q$-Racah polynomials that will be needed to prove (1.21). In Section 6, we shall deduce a special product formula from (5.1) that will establish it as an extension of a product formula for the little $q$-Jacobi polynomials obtained by Koornwinder [13].

\section{Evaluation of an integral}

The orthogonality relation for the Askey-Wilson polynomials defined in (1.20) is

$$
\int_{-1}^{1} w(x ; a, b, c, d) p_{m}(x ; a, b, c, d) p_{n}(x ; a, b, c, d) d x=\delta_{m, n} h_{n}^{-1}
$$

where

$$
\begin{gathered}
w\left(x ; a_{1}, a_{2}, a_{3}, a_{4}\right)=\frac{\left(e^{2 i \theta}, e^{-2 i \theta} ; q\right)_{\infty} \csc \theta}{\prod_{j=1}^{4}\left(a_{j} e^{i \theta}, a_{j} e^{-i \theta} ; q\right)_{\infty}}, \quad x=\cos \theta, \\
h_{n}=\kappa^{-1}(a, b, c, d) \frac{\left(1-a b c d q^{2 n-1}\right)\left(a b c d q^{-1}, a b, a c, a d ; q\right)_{n}}{\left(1-a b c d q^{-1}\right)(q, c d, b d, b c ; q)_{n}} a^{-2 n} \\
\kappa(a, b, c, d)=\frac{2 \pi(a b c d ; q)_{\infty}}{(q, a b, a c, a d, b c, b d, c d ; q)_{\infty}}
\end{gathered}
$$

where $\max (|a|,|b|,|c|,|d|)<1$; see [3] and [8].

Let $k, m$, and $r$ be three nonnegative integers and let

$$
\begin{aligned}
S_{r}(m, m+k):= & \int_{-1}^{1} w(x ; a, b, c, d)\left(d e^{i \theta}, d e^{-i \theta}\right)_{r} \\
& \times p_{m}(x ; a, b, c, d) p_{m+k}(x ; a, b, c, d) d x .
\end{aligned}
$$

It is clear from (2.1) that this integral will vanish unless $r \geq k$. We will show in this section that

$$
\begin{gathered}
S_{r}(m, m+k)=h_{m}^{-1} \frac{\left(q^{-r}, b c q^{m}, q^{m+1} ; q\right)_{k}\left(a d q^{m}, b d q^{m}, c d q^{m} ; q\right)_{r}}{\left(q, a d q^{m} ; q\right)_{k}\left(a b c d q^{2 m} ; q\right)_{k+r}} \\
\times \frac{\left(a b c d q^{m+k-1} ; q\right)_{m}}{\left(a b c d q^{m+r-1} ; q\right)_{m}}\left(a d q^{r}\right)^{k}{ }_{10} W_{9}\left(q^{1-2 m-r} / a b c d ; q^{1-2 m-k-r} / a b c d, q^{k-r}\right. \\
\left.q^{-r}, q^{1-m} / a b, q^{1-m} / a c, q^{1-m} / b c, q^{-m} ; q, q^{2} / d^{2}\right)
\end{gathered}
$$

for $m=0,1,2, \ldots$, and $r=k, k+1, k+2, \ldots$, where $k=0,1,2, \ldots$ There is no particular reason for attaching the parameter $d$ to the $r$-shifted factorials inside the integral in (2.5). It could be any one of the four parameters $a, b, c, d$. The choice of this parameter will naturally affect the symmetry property of the right-hand side of (2.6). Since, by Sears transformation formula [8, (III. 15)]

$$
p_{m+k}(x ; a, b, c, d)=\frac{(b c, b d ; q)_{m+k}}{(a c, a d ; q)_{m+k}}\left(\frac{a}{b}\right)^{m+k} p_{m+k}(x ; b, a, c, d),
$$


we find, by using (2.1), that

$$
S_{r}(m, m+k)=\kappa(a, b, c, d) \frac{(b c, b d ; q)_{m+k}(a d, b d, c d ; q)_{r}}{(a c, a d ; q)_{m+k}(a b c d ; q)_{r}}\left(\frac{a}{b}\right)^{m+k} K_{r}
$$

where

$$
\begin{aligned}
K_{r}= & \sum_{s=0}^{m+k} \frac{\left(q^{-m-k}, a b c d q^{m+k-1}, b d q^{r} ; q\right)_{s}}{\left(q, a b c d q^{r}, b d ; q\right)_{s}} q^{s} \\
& \times{ }_{4} \varphi_{3}\left[\begin{array}{c}
q^{-m}, a b c d q^{m-1}, a d q^{r}, a b q^{s} \\
a b c d q^{r+s}, a d, a b
\end{array} ; q, q\right] \\
= & \frac{\left(a d q^{r}, a b c d q^{m-1} ; q\right)_{m}}{(a b, a d ; q)_{m}}(-1)^{m} q^{-\left(\begin{array}{c}
m \\
2
\end{array}\right)} \\
& \times \sum_{s=0}^{m+k} \frac{\left(q^{-m-k}, a b c d q^{m+k-1}, b d q^{r} ; q\right)_{s}\left(a b q^{s} ; q\right)_{m}}{\left(q, a b c d q^{r}, b d ; q\right)_{s}\left(a b c d q^{r+s} ; q\right)_{m}} q^{s} \\
& \times{ }_{4} \varphi_{3}\left[\begin{array}{c}
q^{-m}, q^{1-m-r-s} / a b c d, q^{1-m} / a b, q^{1-m} / a d \\
q^{2-2 m} / a b c d, q^{1-m-r} / a d, q^{1-m-s} / a b
\end{array} ;, q\right],
\end{aligned}
$$

the second ${ }_{4} \varphi_{3}$ on the right having been obtained from the first by simply reversing the sum. By Watson's transformation formula [8, (III. 17)],

$$
\begin{aligned}
& { }_{4} \varphi_{3}\left[\begin{array}{c}
q^{-m}, q^{1-m-r-s} / a b c d, q^{1-m} / a b, q^{1-m} / a d \\
q^{2-2 m} / a b c d, q^{1-m-r} / a d, q^{1-m-s} / a b
\end{array} ;, q\right] \\
& =\frac{\left(q^{1-m-r} / c d, q^{1-m+s} ; q\right)_{m}}{\left(q^{2-2 m-r} / a b c d, a b q^{s} ; q\right)_{m}}{ }_{8} W_{7}\left(q^{1-2 m-r} / a b c d\right. \text {; } \\
& \left.q^{1-m} / a b, q^{1-m} / b c, q^{-m}, q^{-r}, q^{1-m-r-s} / a b c d ; q, b q^{s+1} / d\right) \text {. }
\end{aligned}
$$

So the series over $s$ in the last line on the right-hand side of (2.9) becomes

$$
\begin{aligned}
& \frac{\left(q^{1-m-r} / c d ; q\right)_{m}}{\left(q^{2-2 m-r} / a b c d, a b c d q^{r} ; q\right)_{m}} \sum_{j=0}^{r} \frac{1-q^{1-2 m-r+2 j} / a b c d}{1-q^{1-2 m-r} / a b c d} \\
& \quad \times \frac{\left(q^{1-2 m-r} / a b c d, q^{1-m} / a b, q^{1-m} / b c, q^{-m}, q^{-r} ; q\right)_{j}}{\left(q, q^{1-m-r} / c d, q^{1-m-r} / a d, q^{2-m-r} / a b c d, q^{2-2 m} / a b c d ; q\right)_{j}}\left(\frac{b q}{d}\right)^{j} \\
& \times \sum_{s=m-j}^{m+k} \frac{\left(q^{-m-k}, a b c d q^{m+k-1}, b d q^{r} ; q\right)_{s}\left(q^{1-m-r-s} / a b c d ; q\right)_{j}\left(q^{1-m+s+j} ; q\right)_{m-j}}{\left(q, a b c d q^{r+m}, b d ; q\right)_{s}} q^{s(j+1)} \\
& =\frac{\left(q^{1-m-r} / c d ; q\right)_{m}}{\left(q^{2-2 m-r} / a b c d, a b c d q^{r} ; q\right)_{m}} \sum_{j=0}^{r} \frac{1-q^{1-2 m-r+2 j} / a b c d}{1-q^{1-2 m-r} / a b c d} \\
& \quad \times \frac{\left(q^{1-2 m-r} / a b c d, q^{1-m} / a b, q^{1-m} / b c, q^{-m}, q^{-r} ; q\right)_{j}}{\left(q, q^{1-m-r} / c d, q^{1-m-r} / a d, q^{2-m-r} / a b c d, q^{2-2 m} / a b c d ; q\right)_{j}}\left(\frac{b q}{d}\right)^{j} \\
& \quad \times \frac{\left(q^{-m-k}, a b c d q^{m+k-1}, b d q^{r} ; q\right)_{m-j}\left(q^{1-2 m-r+j} / a b c d ; q\right)_{j}}{\left(a b c d q^{m+r}, b d ; q\right)_{m-j}} q^{(j+1)(m-j)} \\
& \quad \times{ }_{3} \varphi_{2}\left[\begin{array}{c}
q^{-j-k}, a b c d q^{2 m+k-j-1}, b d q^{m-j+r} \\
a b c d q^{2 m-2 j+r}, b d q^{m-j}
\end{array} ;, q\right] .
\end{aligned}
$$


Summing the balanced $\varphi_{2}$ series above by the $q$-Saalschütz formula [8, (II.12)] and simplifying, we find that the expression in (2.11) is

$$
\begin{aligned}
& \frac{\left(q^{-r}, a c q^{m} ; q\right)_{k}\left(q^{1-m-r} / c d, q^{-m-k}, a b c d q^{m+k-1}, b d q^{r} ; q\right)_{m}}{\left(q^{2-2 m-r} / a b c d, a b c d q^{r} ; q\right)_{m}\left(b d, a b c d q^{m+r} ; q\right)_{m+k}} \\
& \times{ }_{10} W_{9}\left(q^{1-2 m-r} / a b c d ; q^{1-2 m-r-k} / a b c d, q^{k-r}, q^{-r}, q^{1-m} / a b\right. \\
& \left.q^{1-m} / a c, q^{1-m} / b c, q^{-m} ; q, q^{2} / d^{2}\right) .
\end{aligned}
$$

Substitution in (2.9), followed by another round of simplifications, completes the proof of (2.6).

\section{Proof of the addition formula (1.16)}

We now can multiply (2.6) by an arbitrary sequence $\left\{\mu_{r}\right\}_{r=0}^{\infty}$ and sum over $r$ to get a general integration formula, but the most interesting of these is the one that produces the $q$-Legendre-type polynomials

$$
p_{n}(x ; d, q / d, b, q / b)={ }_{4} \varphi_{3}\left[\begin{array}{c}
q^{-n}, q^{n+1}, d e^{i \theta}, d e^{-i \theta} \\
q, b d, d q / b
\end{array} ; q, q\right]
$$

as part of the integrand. Denoting

$$
\begin{aligned}
I_{n, m, m+k}:=\int_{-1}^{1} w & (x ; a, b, c, d) p_{n}(x ; d, q / d, b, q / b) \\
& \times p_{m}(x ; a, b, c, d) p_{m+k}(x ; a, b, c, d) d x
\end{aligned}
$$

we have, by (2.6),

$$
\begin{aligned}
& I_{n, m, m+k}= \sum_{r=0}^{n} \frac{\left(q^{-n}, q^{n+1} ; q\right)_{r} S_{r}(m, m+k)}{(q, q, b d, d q / b ; q)_{r}} q^{r} \\
&= h_{m}^{-1} \frac{\left(q^{-n}, q^{n+1}, b c q^{m}, b d q^{m}, c d q^{m}, q^{m+1} ; q\right)_{k}}{(q, q, b d, d q / b ; q)_{k}\left(a b c d q^{2 m} ; q\right)_{2 k}}(-a d)^{k} q^{\left(\begin{array}{c}
k+1 \\
2
\end{array}\right)} \\
& \quad \times \sum_{r=0}^{n-k} \frac{\left(q^{k-n}, q^{k+n+1}, a d q^{m+k}, b d q^{m+k}, c d q^{m+k}, a b c d q^{m+k-1} ; q\right)_{r}}{\left(q, q^{k+1}, b d q^{k}, d q^{k+1} / b, a b c d q^{2 m+2 k}, a b c d q^{2 m+k-1} ; q\right)_{r}} q^{r} \\
& \quad \times{ }_{10} W_{9}\left(q^{1-2 m-k-r} / a b c d ; q^{1-2 m-r-2 k} / a b c d, q^{-k-r},\right. \\
&\left.q^{-r}, q^{1-m} / a b, q^{1-m} / a c, q^{1-m} / b c, q^{-m} ; q, q^{2} / d^{2}\right) .
\end{aligned}
$$

The interesting thing about it is that we now may identify the parameters with those of the product formula $[8,(8.2 .6)]$ :

$$
\begin{gathered}
{ }_{4} \varphi_{3}\left[\begin{array}{c}
q^{-n}, A q^{n}, b_{1}, b_{2} \\
B, b_{3}, q A b_{1} b_{2} / B b_{3}
\end{array} ; q, q\right]{ }_{4} \varphi_{3}\left[\begin{array}{c}
q^{-n}, A q^{n}, c_{1}, c_{2} \\
A q / B, A q / b_{3}, B b_{3} c_{1} c_{2} / A q
\end{array} ; q, q\right] \\
=\sum_{r=0}^{n} \frac{\left(q^{-n}, A q^{n}, c_{1}, c_{2}, q A b_{1} / B b_{3}, q A b_{2} / B b_{3} ; q\right)_{r}}{\left(q, A q / B, A q / b_{3}, A q / B b_{3}, q A b_{1} b_{2} / B b_{3}, B b_{3} c_{1} c_{2} / A q ; q\right)_{r}} q^{r} \\
\times{ }_{10} W_{9}\left(B b_{3} q^{-r-1} / A ; B q^{-r} / A, b_{3} q^{-r} / A, q^{-r}, b_{1}, b_{2}\right. \\
\left.B b_{3} c_{1} / A q, B b_{3} c_{2} / A q ; q, \frac{A q^{2}}{b_{1} b_{2} c_{1} c_{2}}\right) .
\end{gathered}
$$


This formula is valid even if $n$ is not an integer as long as the parameters $b_{1}, b_{2}, c_{1}, c_{2}$ are such that both of the ${ }_{4} \varphi_{3}$ series on the left-hand side and the series on the righthand side terminate. We are reproducing this formula here instead of just referring the reader to [8] because of its crucial nature as far as this paper is concerned. Note the relationship between the product of $q^{-n}$ and $A q^{n}$, and the ratio of $q$ times $B b_{3} / A q$ to the product of the three coefficients of $q^{-r}$ in the ${ }_{10} W_{9}$ series. These two numbers are the same. When $k=0$, this number for the ${ }_{10} W_{9}$ series in (3.3) is $q$. This is why this procedure produces an addition formula only for the $q$-Legendre-type polynomials. So, by (3.4), we now have

$$
\begin{aligned}
I_{n, m, m+k}=h_{m}^{-1} & \frac{\left(q^{-n}, q^{n+1}, b c q^{m}, b d q^{m}, c d q^{m}, q^{m+1} ; q\right)_{k}}{(q, q, b d, d q / b ; q)_{k}\left(a b c d q^{2 m} ; q\right)_{2 k}}(-a d)^{k} q_{2}^{\left(\begin{array}{c}
k+1 \\
2
\end{array}\right)} \\
& \times{ }_{4} \varphi_{3}\left[\begin{array}{c}
q^{k-n}, q^{k+n+1}, q^{-m}, q^{1-m} / a c \\
q^{k+1}, b d q^{k}, q^{2-2 m} / a b c d
\end{array} ;, q\right] \\
& \times{ }_{4} \varphi_{3}\left[\begin{array}{c}
q^{k-n}, q^{k+n+1}, a d q^{m+k}, c d q^{m+k} \\
q^{k+1}, d q^{k+1} / b, a b c d q^{2 m+2 k} ; q, q
\end{array}\right] .
\end{aligned}
$$

For $k=0$, we have a product formula:

$$
\begin{gathered}
\int_{-1}^{1} w(x ; a, b, c, d) p_{n}(x ; d, q / d, b, q / b) p_{m}^{2}(x ; a, b, c, d) d x \\
=h_{m}^{-1}{ }_{4} \varphi_{3}\left[\begin{array}{c}
q^{-n}, q^{n+1}, q^{-m}, q^{1-m} / a c \\
q, b d, q^{2-2 m} / a b c d
\end{array}\right] \\
\left.\quad \times{ }_{4} \varphi_{3}\left[\begin{array}{c}
q^{-n}, q^{n+1}, a d q^{m}, c d q^{m} \\
q, d q / b, a b c d q^{2 m}
\end{array}\right] q, q\right] .
\end{gathered}
$$

This suggests an expression of the form (1.16). To find the coefficient $A_{0}$, we multiply (1.16) by $p_{m}(x ; a, b, c, d) w(x ; a, b, c, d)$ and integrate over $x$ from -1 to 1 . This gives (1.17) after having used (3.6) and the transformation

$$
\begin{aligned}
& { }_{4} \varphi_{3}\left[\begin{array}{c}
q^{-n}, q^{n+1}, q^{-m}, q^{1-m} / a c \\
q, b d, q^{2-2 m} / a b c d
\end{array} ; q, q\right]= \\
& \frac{\left(a b c d q^{2 m} ; q\right)_{n}}{\left(q^{2-2 m} / a b c d ; q\right)_{n}}\left(q^{1-2 m} / a b c d\right)^{n}{ }_{4} \varphi_{3}\left[\begin{array}{c}
q^{-n}, q^{n+1}, b d q^{m}, a b c d q^{m-1} \\
q, b d, a b c d q^{2 m}
\end{array} ; q, q\right] \text {. }
\end{aligned}
$$

To obtain $A_{k}$, we multiply $(1.16)$ by $p_{m+k}(x ; a, b, c, d) w(x ; a, b, c, d)$, integrate, and use (3.5). This gives (1.18). Finally, (1.19) for $A_{k}^{\prime}$ is obtained by multiplying (1.16) by $p_{m-k}(x ; a, b, c, d) w(x ; a, b, c, d)$, integrating, and then using (3.5) after having replaced $m$ by $m-k$. It is assumed, of course, that a formula similar to (3.7) has been used in both cases to reach the final form of the coefficients as given in (1.18) and (1.19). 
If we set $c=0$ in (1.16)-(1.19), we obtain the following addition formula

$$
\begin{aligned}
& p_{n}(x ; d, q / d, b, q / b)_{3} \varphi_{2}\left[\begin{array}{c}
q^{-m}, a e^{i \theta}, a e^{-i \theta} \\
a b, a d
\end{array} ; q, q\right]= \\
& (-1)^{n} q^{-\left(\begin{array}{c}
n+1 \\
2
\end{array}\right)_{3} \varphi_{2}}\left[\begin{array}{c}
q^{-n}, q^{n+1}, a d q^{m} \\
q, d q / b
\end{array} ; q, q\right]{ }_{3} \varphi_{2}\left[\begin{array}{c}
q^{-n}, q^{n+1}, b d q^{m} \\
q, b d
\end{array} ; q, q\right] \\
& \times{ }_{3} \varphi_{2}\left[\begin{array}{c}
q^{-m}, a e^{i \theta}, a e^{-i \theta} \\
a b, a d
\end{array} ; q, q\right] \\
& +\sum_{k=1}^{n} \frac{\left(a b q^{m}, a d q^{m}, q^{-n}, q^{n+1} ; q\right)_{k}}{(q, q, b d, d q / b ; q)_{k}}(-1)^{n} q^{k(k+1)-\left(\begin{array}{c}
n+1 \\
2
\end{array}\right)}(d / a)^{k} \\
& \times{ }_{3} \varphi_{2}\left[\begin{array}{c}
q^{k-n}, q^{n+k+1}, a d q^{m+k} \\
q^{k+1}, d q^{k+1} / b
\end{array} ; q, q\right]{ }_{3} \varphi_{2}\left[\begin{array}{c}
q^{k-n}, q^{k+n+1}, b d q^{m+k} \\
q^{k+1}, b d q^{k}
\end{array} ; q, q\right] \\
& \times{ }_{3} \varphi_{2}\left[\begin{array}{c}
q^{-m-k}, a e^{i \theta}, a e^{-i \theta} \\
a b, a d
\end{array} ; q, q\right] \\
& +\sum_{k=1}^{\min (m, n)} \frac{\left(q^{-m}, q^{1-m} / b d, q^{-n}, q^{n+1} ; q\right)_{k}}{(q, q, b d, d q / b ; q)_{k}}(-1)^{n} q^{k-\left(\begin{array}{c}
n+1 \\
2
\end{array}\right)}\left(a b d^{2} q^{2 m}\right)^{k} \\
& \times{ }_{3} \varphi_{2}\left[\begin{array}{c}
q^{k-n}, q^{n+k+1}, a d q^{m} \\
q^{k+1}, d q^{k+1} / b
\end{array} ; q, q\right]{ }_{3} \varphi_{2}\left[\begin{array}{c}
q^{k-n}, q^{k+n+1}, b d q^{m} \\
q^{k+1}, b d q^{k}
\end{array} ; q, q\right] \\
& \times{ }_{3} \varphi_{2}\left[\begin{array}{c}
q^{-m+k}, a e^{i \theta}, a e^{-i \theta} \\
a b, a d
\end{array} ; q, q\right],
\end{aligned}
$$

which is essentially the same as Theorem 5.1 of [10].

\section{Evaluation of a series}

In this section, we shall compute the sum

$$
\begin{aligned}
J_{\nu, m, m+k}:= & \sum_{z=0}^{N} \rho_{N}(z ; a, b, c)_{4} \varphi_{3}\left[\begin{array}{c}
q^{-\nu}, q^{\nu+1}, q^{z-N}, c^{-1} q^{-z} \\
q, q^{-N}, q / c
\end{array}\right] \\
& \times R_{m}\left(q^{z} ; a, b, c, q^{-N}\right) R_{m+k}\left(q^{z} ; a, b, c, q^{-N}\right)
\end{aligned}
$$

where $\nu$ is a complex parameter, and

$$
\rho_{N}(z ; a, b, c)=\frac{\left(1-c^{2 z-N}\right)}{\left(1-c q^{-N}\right)} \frac{\left(c q^{-N}, a q, b c q, q^{-N} ; q\right)_{z}}{\left(q, c q^{-N} / a, q^{-N} / b, c q ; q\right)_{z}}(a b q)^{-z}
$$

is the weight function associated with the orthogonality relation

$$
\sum_{z=0}^{N} \rho_{N}(z ; a, b, c) R_{m}\left(q^{z} ; a, b, c, q^{-N}\right) R_{n}\left(q^{z} ; a, b, c, q^{-N}\right)=\delta_{m, n} / k_{n}
$$

where

$$
k_{n}=\frac{(b q, a q / c ; q)_{N}}{\left(c^{-1}, a b q^{2} ; q\right)_{N}} \frac{\left(1-a b q^{2 n+1}\right)\left(a b q, a q, b c q, q^{-N} ; q\right)_{n}}{(1-a b q)\left(q, b q, a q / c, a b q^{N+2} ; q\right)_{n}}\left(c q^{-N}\right)^{-n}
$$


see $[8]$.

First, we will show that

$$
\begin{aligned}
T_{r}(m, m+k):= & \sum_{z=0}^{N} \rho_{N}(z ; a, b, c)\left(q^{z-N}, c^{-1} q^{-z} ; q\right)_{r} \\
& \times R_{m}\left(q^{z} ; a, b, c, q^{-N}\right) R_{m+k}\left(q^{z} ; a, b, c, q^{-N}\right)
\end{aligned}
$$

is zero if $0 \leq r<k$ and is given by

$$
\begin{aligned}
& T_{r}(m, m+k) \\
& =k_{m}^{-1} \frac{\left(q^{-r}, q^{m+1}, a q^{m+1} ; q\right)_{k}\left(q^{m-N}, a q^{m+1} / c, b q^{m+1} ; q\right)_{r}\left(a b q^{m+k+1} ; q\right)_{m}}{\left(q, q^{m-N} ; q\right)_{k}\left(a b q^{2 m+2} ; q\right)_{k+r}\left(a b q^{m+r+1} ; q\right)_{m}} q^{(r-N) k} \\
& \times{ }_{10} W_{9}\left(q^{-2 m-r-1} / a b ; q^{-2 m-k-r-1} / a b\right. \text {, } \\
& \left.q^{k-r}, q^{-r}, q^{-m} / a, q^{-m} / b c, q^{-N-m-1} / a b, q^{-m} ; q, c q^{N+2}\right),
\end{aligned}
$$

for $r=k, k+1, \ldots, N$.

Using [8, (III.15)] on the two $R$ series in (4.5) followed by the use of [8, (II.21)], we find that

$$
\begin{aligned}
T_{r}(m, m+k)=\frac{\left(a b q^{2}, c^{-1} ; q\right)_{N}\left(a q / c, a b q^{N+2} ; q\right)_{m}\left(b q, a b q^{N+2} ; q\right)_{m+k}}{(b q, a q / c ; q)_{N}\left(b c q, q^{-N} ; q\right)_{m}\left(a q, q^{-N} ; q\right)_{m+k}} \\
\quad \times \frac{\left(q^{-N}, a q / c, b q ; q\right)_{r}}{\left(a b q^{2} ; q\right)_{r}}\left(\frac{a b q^{2 N+2}}{c}\right)^{-m}\left(b q^{N+1}\right)^{-k} \\
\quad \times \sum_{s=0}^{m+k} \frac{\left(q^{-m-k}, a b q^{m+k+1}, b q^{r+1} ; q\right)_{s}}{\left(q, a b q^{r+2}, b q ; q\right)_{s}} q^{s} \\
\times{ }_{4} \varphi_{3}\left[\begin{array}{c}
q^{-m}, a b q^{m+1}, a q^{r+1} / c, a b q^{N+s+2} \\
a b q^{r+s+2}, a q / c, a b q^{N+2}
\end{array} ;, q\right] .
\end{aligned}
$$

The sum over $s$ in (4.7) can be transformed, as in Section 2, by first reversing the order of summation of the ${ }_{4} \varphi_{3}$ series and then using [8, (III.17)], to get

$$
\begin{aligned}
& \frac{\left(a b q^{m+1}, a q^{r+1} / c, q^{r-N} ; q\right)_{m}}{\left(a b q^{N+2}, a q / c, a b q^{m+r+1} ; q\right)_{m}}\left(a b q^{N+1}\right)^{m}(-1)^{m} q^{\left(\begin{array}{c}
m+1 \\
2
\end{array}\right)} \\
& \quad \times \sum_{s=0}^{m+k} \frac{\left(q^{-m-k}, a b q^{m+k+1}, b q^{r+1} ; q\right)_{s}}{\left(q, a b q^{r+2}, b q ; q\right)_{s}} \frac{\left(q^{s+1-m} ; q\right)_{m}}{\left(a b q^{r+s+2} ; q\right)_{m}} q^{s} \\
& \quad \times{ }_{8} W_{7}\left(q^{-2 m-r-1} / a b ; q^{-m}, q^{-m} / b c, q^{-N-m-1} / a b, q^{-r}\right. \\
& = \\
& \quad \frac{\left(a b q^{m+1}, a q^{r+1} / c, q^{r-N} ; q\right)_{m}}{\left(a b q^{N+2}, a q / c, a b q^{m+r+1} ; q\right)_{m}}\left(a b q^{N+1}\right)^{m}(-1)^{m} q^{\left(\begin{array}{c}
m+1 \\
2
\end{array}\right)} \\
& \quad \times \sum_{j=0}^{\min (m, r)} \frac{1-q^{2 j-2 m-r-1} / a b}{1-q^{-2 m-r-1} / a b} \frac{\left(q^{-2 m-r-1} / a b, q^{-m}, q^{-m} / b c, q^{-N-m-1} / a b, q^{-r} ; q\right)_{j}}{\left(q, q^{-m-r} / a b, c q^{-m-r} / a, q^{N+1-m-r}, q^{-2 m} / a b ; q\right)_{j}} \times
\end{aligned}
$$




$$
\begin{aligned}
& \times \frac{\left(q^{-m-k}, a b q^{m+k+1}, b q^{r+1} ; q\right)_{m-j}\left(q^{j-2 m-r-1} / a b ; q\right)_{j}}{(b q ; q)_{m-j}\left(a b q^{r+2} ; q\right)_{2 m-j}}\left(b c q^{N+2}\right)^{j} \\
& \times{ }_{3} \varphi_{2}\left[\begin{array}{c}
q^{-j-k}, a b q^{2 m-j+k+1}, b q^{m-j+r+1} \\
a b q^{2 m-2 j+r+2}, b q^{m-j+1}
\end{array} ; q, q\right],
\end{aligned}
$$

which, on using [8, (II. 12)] and simplifying the coefficients, becomes

$$
\begin{gathered}
\frac{\left(a b q^{m+1}, a q^{r+1} / c, q^{r-N}, q^{k+1}, a b q^{m+k+1}, b q^{r+1} ; q\right)_{m}}{\left(a b q^{N+2}, a q / c, a b q^{m+r+1} ; q\right)_{m}(b q ; q)_{m+k}\left(a b q^{r+2} ; q\right)_{2 m+k}}\left(a b q^{N+2}\right)^{m}\left(b q^{r+1}\right)^{k} \\
\times{ }_{10} W_{9}\left(q^{-2 m-r-1} / a b ; q^{-2 m-r-k-1} / a b, q^{-m}\right. \\
\left.q^{-m} / b c, q^{-m} / a, q^{-r}, q^{k-r}, q^{-N-m-1} / a b ; q, c q^{N+2}\right)
\end{gathered}
$$

for $r=k, k+1, \ldots, N$ and is 0 if $0 \leq r<k$. Substitution of this into (4.7) completes the proof of (4.6). This leads to the following expression:

$$
\begin{aligned}
& J_{\nu, m, m+k}= \\
& k_{m}^{-1} \frac{\left(q^{-\nu}, q^{\nu+1}, a q^{m+1}, b q^{m+1}, a q^{m+1} / c, q^{m+1} ; q\right)_{k}}{\left(q, q, q^{-N}, q / c ; q\right)_{k}\left(a b q^{2 m+2} ; q\right)_{2 k}}(-1)^{k} q^{-N k+\left(\begin{array}{c}
k+1 \\
2
\end{array}\right)} \\
& \times \sum_{r=0}^{N-m-k} \frac{\left(q^{k-\nu}, q^{k+\nu+1}, q^{m+k-N}, a q^{m+k+1} / c, b q^{m+k+1}, a b q^{m+k+1} ; q\right)_{r}}{\left(q, q^{k+1}, a b q^{2 m+2 k+2}, a b q^{2 m+k+1}, q^{k-N}, q^{k+1} / c ; q\right)_{r}} q^{r} \\
& \times{ }_{10} W_{9}\left(q^{-2 m-k-r-1} / a b ; q^{-2 m-r-k-1} / a b, q^{-m}, q^{-m} / b c, q^{-m} / a, q^{-r}, q^{-k-r}\right. \text {, } \\
& \left.q^{-N-m-1} / a b ; q, c q^{N+2}\right) \\
& =k_{m}^{-1} \frac{\left(q^{-\nu}, q^{\nu+1}, a q^{m+1}, b q^{m+1}, a q^{m+1} / c, q^{m+1} ; q\right)_{k}}{\left(q, q, q^{-N}, q / c ; q\right)_{k}\left(a b q^{2 m+2} ; q\right)_{2 k}}(-1)^{k} q^{-N k+\left(\begin{array}{c}
k+1 \\
2
\end{array}\right)} \\
& \times{ }_{4} \varphi_{3}\left[\begin{array}{c}
q^{k-\nu}, q^{k+\nu+1}, q^{-m} / a, q^{-m} / b c \\
q^{k+1}, q^{k+1} / c, q^{-2 m} / a b
\end{array} q, q\right] \\
& \times{ }_{4} \varphi_{3}\left[\begin{array}{c}
q^{k-\nu}, q^{k+\nu+1}, q^{m+k-N}, a b q^{m+k+1} \\
q^{k+1}, q^{k-N}, a b q^{2 m+2 k+2} ; q, q
\end{array}\right],
\end{aligned}
$$

by (3.4), provided the first ${ }_{4} \varphi_{3}$ terminates as does the second one because of the numerator parameter $q^{m+k-N}$. This requires us to assume that the parameters $a, b, c$ are such that either $q^{-m} / a$, or $q^{-m} / b c$ is of the form $q^{-l}$ where $l$ is a nonnegative integer. This we shall assume to be true in the following sections.

\section{Proof of the addition formula (1.21)}

As in (3.6), we find from (4.8) that

$$
\begin{aligned}
& \sum_{z=0}^{N} \rho_{N}(z ; a, b, c)_{4} \varphi_{3}\left[\begin{array}{c}
q^{-\nu}, q^{\nu+1}, q^{z-N}, c^{-1} q^{-z} \\
q, q^{-N}, q / c
\end{array} ; q, q\right] R_{m}^{2}\left(q^{z} ; a, b, c, q^{-N}\right)= \\
& k_{m}^{-1}{ }_{4} \varphi_{3}\left[\begin{array}{c}
q^{-\nu}, q^{\nu+1}, q^{-m} / a, q^{-m} / b c \\
q, q / c, q^{-2 m} / a b
\end{array} ; q, q\right]{ }_{4} \varphi_{3}\left[\begin{array}{c}
q^{-\nu}, q^{\nu+1}, q^{m-N}, a b q^{m+1} \\
q, q^{-N}, a b q^{2 m+2}
\end{array} ; q, q\right] .
\end{aligned}
$$


This, of course, suggests an expansion of the form (1.21). To find the coefficient $B_{0}$, we multiply both sides of (1.21) by $R_{m}\left(q^{z} ; a, b, c, q^{-N}\right)$ and use (4.3) and (5.1) to get (1.23). To compute $B_{k}$, we multiply (1.21) by $R_{m+k}\left(q^{z} ; a, b, c, q^{-N}\right), k=0,1 \ldots$ and use (4.3) and (4.8) to get (1.24). Finally, the expression (1.25) for $B_{k}^{\prime}$ is obtained by first multiplying (1.21) by $R_{m-k}\left(q^{z} ; a, b, c, q^{-N}\right)$, then using (4.3) and (4.8) (with $m$ replaced by $m-k$ ).

With a view to compare a limiting case of (1.21) with Koornwinder's [13] addition formula for the little $q$-Jacobi polynomials, let us first rewrite (1.21) by replacing $m$ and $b$ by $y$ and $q^{x}$, respectively. We first replace $R_{m \pm k}\left(q^{z} ; a, b, c, q^{-N}\right)$ by

$$
\begin{aligned}
& R_{m \pm k}\left(q^{z} ; a, b, c, q^{-N}\right) \\
& =\frac{(b q, a q / c ; q)_{m \mp k}}{(a q, b c q ; q)_{m \mp k}} c^{m \mp k}{ }_{4} \varphi_{3}\left[\begin{array}{c}
q^{-m \pm k,}, a b q^{m \mp k+1}, q^{z-N}, q^{-z} / c \\
b q, q^{-N}, a q / c
\end{array}\right]
\end{aligned}
$$

(see [7, (III.15)]), so we get

$$
\begin{aligned}
& { }_{4} \varphi_{3}\left[\begin{array}{c}
q^{-\nu}, q^{\nu+1}, q^{z-N}, q^{-z} / c \\
q, q / c, q^{-N}
\end{array} ;, q\right]{ }_{4} \varphi_{3}\left[\begin{array}{c}
q^{-y}, a q^{x+y+1}, q^{z-N}, q^{-z} / c \\
q^{x+1}, a q / c, q^{-N}
\end{array} q, q\right] \\
& ={ }_{4} \varphi_{3}\left[\begin{array}{c}
q^{-\nu}, q^{\nu+1}, q^{-y} / a, q^{-x-y} / c \\
q, q / c, q^{-x-2 y} / a
\end{array} ;, q\right]{ }_{4} \varphi_{3}\left[\begin{array}{c}
q^{-\nu}, q^{\nu+1}, q^{y-N}, a q^{x+y+1} \\
q, a q^{x+2 y+2}, q^{-N}
\end{array} ; q, q\right] \\
& \times{ }_{4} \varphi_{3}\left[\begin{array}{c}
q^{-y}, a q^{x+y+1}, q^{z-N}, q^{-z} / c \\
q^{x+1}, a q / c, q^{-N}
\end{array} ; q, q\right] \\
& +\sum_{k=1}^{\infty} \frac{\left(q^{-\nu}, q^{\nu+1}, a q^{x+y+1}, a q^{y+1}, q^{x+y+1}, a q^{y+1} / c, q^{y-N} ; q\right)_{k}}{\left(q, q, q / c, q^{-N} ; q\right)_{k}\left(a q^{x+2 y+1} ; q\right)_{2 k}}(-1)^{k} q^{\left(\begin{array}{c}
k+1 \\
2
\end{array}\right)} \\
& \times{ }_{4} \varphi_{3}\left[\begin{array}{c}
q^{k-\nu}, q^{k+\nu+1}, q^{-y} / a, q^{-x-y} / c \\
q^{k+1}, q^{k+1} / c, q^{-x-2 y} / a
\end{array} q, q\right] \\
& \times{ }_{4} \varphi_{3}\left[\begin{array}{c}
q^{k-\nu}, q^{k+\nu+1}, q^{k+y-N}, a q^{x+y+k+1} \\
q^{k+1}, a q^{x+2 y+2 k+2}, q^{k-N}
\end{array} ;, q\right] \\
& \times{ }_{4} \varphi_{3}\left[\begin{array}{c}
q^{-y-k}, a q^{x+y+k+1}, q^{z-N}, q^{-z} / c \\
q^{x+1}, a q / c, q^{-N}
\end{array} ;, q\right] \\
& +\sum_{k=1}^{y} \frac{\left(q^{-\nu}, q^{\nu+1}, q^{-y} / a, q^{-y}, q^{-x-y} / c, c q^{-y} / a ; q\right)_{k}}{\left(q, q, q / c, q^{-N} ; q\right)_{k}\left(q^{-x-2 y-1} / a ; q\right)_{2 k}}\left(-c q^{N+x+1}\right)^{-k} q^{\left(\begin{array}{c}
k+1 \\
2
\end{array}\right)} \\
& \times{ }_{4} \varphi_{3}\left[\begin{array}{c}
q^{k-\nu}, q^{k+\nu+1}, q^{k-y} / a, q^{k-x-y} / c \\
q^{k+1}, q^{k+1} / c, q^{2 k-x-2 y} / a
\end{array} q, q\right] \\
& \times{ }_{4} \varphi_{3}\left[\begin{array}{c}
q^{k-\nu}, q^{k+\nu+1}, q^{y-N}, a q^{x+y+1} \\
q^{k+1}, a q^{x+2 y+2}, q^{k-N}
\end{array} ; q, q\right] \\
& \times{ }_{4} \varphi_{3}\left[\begin{array}{c}
q^{k-y}, a q^{x+y-k+1}, q^{z-N}, q^{-z} / c \\
q^{x+1}, a q / c, q^{-N}
\end{array} q, q\right] .
\end{aligned}
$$


Taking the limits $a \rightarrow 0, c \rightarrow \infty$, and $N \rightarrow \infty$ and simplifying the coefficients, we obtain the addition formula (1.26) for the little $q$-Jacobi functions defined in (1.27). Note that the parameters $x$ and $z$ in (1.26) need not be integers, although we need to assume that $q^{x+1}$ and $q^{z+1}$ are both numerically less than 1 for the sake of convergence. The question is whether the requirement of integral $y$ also can be removed by analytic continuation. It seems possible since

$$
\begin{aligned}
& p_{\nu-k}\left(q^{y-k} ; q^{k}, q^{k} \mid q\right) p_{\nu-k}\left(q^{x+y-k} ; q^{k}, q^{k} \mid q\right) \\
& ={ }_{2} \varphi_{1}\left[\begin{array}{c}
q^{k-\nu}, q^{\nu+k+1} \\
q^{k+1} ; q, q^{y-k+1}
\end{array}\right]{ }_{2} \varphi_{1}\left[\begin{array}{c}
q^{k-\nu}, q^{\nu+k+1} \\
q^{k+1}
\end{array} ; q, q^{x+y-k+1}\right] \\
& =\frac{\left(q^{y+1}, q^{y+x+1} ; q\right)_{\infty}}{\left(q^{y+1-k}, q^{y+x+1-k} ; q\right)_{\infty}}{ }_{2} \varphi_{1}\left[\begin{array}{c}
q^{-\nu}, q^{\nu+1} \\
q^{k+1} ; q, q^{y+1}
\end{array}\right]{ }_{2} \varphi_{1}\left[\begin{array}{c}
q^{-\nu}, q^{\nu+1} \\
q^{k+1}
\end{array} ; q, q^{x+y+1}\right] \\
& =\frac{q^{k(k-1)-(x+2 y) k}}{\left(q^{-y}, q^{-x-y} ; q\right)_{k}} p_{\nu}\left(q^{y} ; q^{k}, q^{-k} \mid q\right) p_{\nu}\left(q^{x+y} ; q^{k}, q^{-k} \mid q\right)
\end{aligned}
$$

by $[8,($ III.3)], so that we can rewrite $(1.26)$ in the form

$$
\begin{aligned}
& p_{\nu}\left(q^{z} ; 1,1 \mid q\right) W_{y}\left(q^{z} ; q^{x} \mid q\right) \\
& =p_{\nu}\left(q^{y} ; 1,1 \mid q\right) p_{\nu}\left(q^{x+y} ; 1,1 \mid q\right) W_{y}\left(q^{z} ; q^{x} \mid q\right) \\
& +\sum_{k=1}^{\infty} \frac{\left(q^{-\nu}, q^{\nu+1}, q^{x+y+1} ; q\right)_{k}}{(q, q ; q)_{k}}(-1)^{k} q^{\left.y k+\left({ }_{2}^{k+1}\right)\right)} p_{\nu-k}\left(q^{y} ; q^{k}, q^{k} \mid q\right) \\
& \quad \times p_{\nu-k}\left(q^{x+y} ; q^{k}, q^{k} \mid q\right) W_{y+k}\left(q^{z} ; q^{x} \mid q\right) \\
& +\sum_{k=1}^{\infty} \frac{\left(q^{-\nu}, q^{\nu+1} ; q\right)_{k}}{\left(q, q, q^{-x-y} ; q\right)_{k}} q^{k} p_{\nu}\left(q^{y} ; q^{k}, q^{-k} \mid q\right) \\
& \quad \times p_{\nu}\left(q^{x+y} ; q^{k}, q^{-k} \mid q\right) W_{y-k}\left(q^{z} ; q^{x} \mid q\right),
\end{aligned}
$$

which is free from any divergence problems. It has to be understood, of course, that the second series on the right-hand side will terminate at $k=y$ whenever $y$ is a positive integer. At the time of writing this paper, Erik Koelink has informed us that he has found a more general formula than (5.5) by formal quantum group-theoretic considerations. At the moment, we are not sure how to prove (5.5) directly when $y$ is not a nonnegative integer. Thus, it may be a while longer before the question of an addition formula for the $q$-Legendre functions finally is settled.

\section{A special product formula}

Although the product formula (5.1) is pretty general in having a number of free parameters, it becomes particularly interesting in the special case when $\nu=n$, a nonnegative integer, and the three ${ }_{4} \varphi_{3}$ series on the two sides are the same $q$-Legendre polynomials in three different variables. This is achieved by setting $m=y, a=q^{N-x-y}, b=q^{x-y}$, and $c=q^{-N-1}$. The parameter $x$ has to be a nonnegative integer less than or equal to $N$ since we have stipulated that $q^{-m} / a=q^{x-N}$ is of the form $q^{-n}, n=0,1, \ldots$. In order to avoid singularities, we assume, therefore, that $0 \leq y \leq x$ and $x+y \leq N$. 
Since

$$
\begin{aligned}
& { }_{4} \varphi_{3}\left[\begin{array}{c}
q^{-n}, q^{n+1}, q^{z-N}, q^{-z} / c \\
q, q^{-N}, q / c
\end{array} ; q, q\right]={ }_{4} \varphi_{3}\left[\begin{array}{c}
q^{-n}, q^{n+1}, q^{z-N}, q^{N+1-z} \\
q, q^{-N}, q^{N+2}
\end{array} ; q, q\right] \\
& =R_{n}\left(q^{N-z} ; 1,1, q^{N+1}, q^{-N}\right),
\end{aligned}
$$

which is a $q$-Legendre polynomial of degree $n$ in $q^{N-z}$ as well as a $q$-Legendre polynomial of degree $N-z$ in $q^{n}$, we can rewrite (5.1) in the form

$$
\begin{aligned}
& \sum_{z=0}^{N} \rho_{N}\left(z ; q^{N-x-y}, q^{x-y}, q^{-N-1}\right) \\
& \quad \times R_{y}^{2}\left(q^{z} ; q^{N-x-y}, q^{x-y}, q^{-N-1}, q^{-N}\right) R_{n}\left(q^{N-z} ; 1,1, q^{N+1}, q^{-N}\right) \\
& \quad=k_{y}^{-1} R_{n}\left(q^{N-x} ; 1,1, q^{N+1}, q^{-N}\right) R_{n}\left(q^{N-y} ; 1,1, q^{N+1}, q^{-N}\right) .
\end{aligned}
$$

Using dual orthogonality, we find that

$$
\begin{aligned}
& \sum_{n=0}^{N} \frac{q^{-n}-q^{n+1}}{1-q} R_{n}\left(q^{N-x} ; 1,1, q^{N+1}, q^{-N}\right) \\
& \quad \times R_{n}\left(q^{N-y} ; 1,1, q^{N+1}, q^{-N}\right) R_{n}\left(q^{N-z} ; 1,1, q^{N+1}, q^{-N}\right) \\
& =k_{y} \rho_{N}\left(z ; q^{N-x-y}, q^{x-y}, q^{-N-1}\right) \frac{\left(1-q^{N+1}\right)\left(1-q^{-1-N}\right)}{\left(1-q^{2-2 z+1}\right)\left(1-q^{-1}\right)} \\
& \times R_{y}^{2}\left(q^{z} ; q^{N-x-y}, q^{x-y}, q^{-N-1}, q^{-N}\right) .
\end{aligned}
$$

Use of $[8,($ III.15)] gives

$$
\begin{aligned}
R_{y}\left(q^{z} ; q^{N-x-y}, q^{x-y}, q^{-N-1}, q^{-N}\right)={ }_{4} \varphi_{3}\left[\begin{array}{c}
q^{-y}, q^{-z}, q^{N+1-y}, q^{z-2 N-1} \\
\left.q^{N+1-x-y}, q^{-N}, q^{x-y-N} ; q, q\right]
\end{array}\right] \\
=\frac{\left(q^{N+1-y-z}, q^{N+1-x-z} ; q\right)_{z}}{\left(q^{-N}, q^{-N+x-y} ; q\right)_{z}} q^{z(x+z-2 N-1)} \\
\quad \times{ }_{4} \varphi_{3}\left[\begin{array}{c}
q^{-x}, q^{-y}, q^{-z}, q^{3 N-x-y-z+2} \\
q^{N+1-x-y}, q^{N+1-y-z}, q^{N+1-z-x}
\end{array} ; q, q\right] .
\end{aligned}
$$

Substituting (6.4) into (6.3) and simplifying the coefficient on the right-hand side by using (4.2) and (4.4), we find that

$$
\begin{aligned}
\sum_{n=0}^{N} \frac{q^{-n}-q^{n+1}}{1-q} R_{n}\left(q^{N-x} ; 1,1, q^{N+1}, q^{-N}\right) \\
\times R_{n}\left(q^{N-y} ; 1,1, q^{N+1}, q^{-N}\right) R_{n}\left(q^{N-z} ; 1,1, q^{N+1}, q^{-N}\right) \\
=\frac{\left(q^{x+1}, q^{y+1}, q^{z+1}, q^{2 N+2-x}, q^{2 N+2-y}, q^{2 N+2-z} ; q\right)_{\infty}}{\left(q, q, q^{N+1+x-y-z}, q^{N+1+y-x-z}, q^{N+1+z-x-y}, q^{3 N+2-x-y-z} ; q\right)_{\infty}} \\
\quad \times \frac{\left(1-q^{N+1}\right)^{3}}{1-q} q^{x y+y z+z x} K_{N}\left(q^{x}, q^{y}, q^{z}\right),
\end{aligned}
$$


where

$$
\begin{aligned}
& K_{N}\left(q^{x}, q^{y}, q^{z}\right)=\left(\frac{\left(q^{N+1-x-y}, q^{N+1-y-z}, q^{N+1-z-x} ; q\right)_{\infty}}{\left(q^{N+1-x}, q^{N+1-y}, q^{N+1-z} ; q\right)_{\infty}}\right. \\
& \left.\times{ }_{4} \varphi_{3}\left[\begin{array}{c}
q^{-x}, q^{-y}, q^{-z}, q^{3 N-x-y-z+2} \\
q^{N+1-x-y}, q^{N+1-y-z}, q^{N+1-z-x}
\end{array} ; q, q\right]\right)^{2} .
\end{aligned}
$$

If we take the limit $N \rightarrow \infty$, then the right-hand side of (6.5) corresponds to Koornwinder's kernel $[13,(9.10)]$ for the little $q$-Jacobi polynomials.

Acknowledgement. We would like to thank Erik Koelink for pointing out some references and for comments that helped prepare this final version. This work was supported in part by NSERC grant A6197.

\section{References}

1. R. Askey, Orthogonal Polynomials and Special Functions, CBMS-NSF Regional Conference Series, Applied Math. 21, SIAM, Philadelphia, PA, 1975.

2. R. Askey and J. A. Wilson, A set of orthogonal polynomials that generalize the Racah coefficients or 6-j symbols, SIAM J. Math. Anal. 10, (1979), 1008-1016.

3. - Some Basic Hypergeometric Orthogonal Polynomials That Generalize Jacobi Polynomials, Memoirs Amer. Math. Soc. Number 319, AMS, Providence, RI, 1985.

4. C. F. Dunkl, An addition theorem for some $q$-Hahn polynomials, Monatsh. Math. 85 (1977), 5-37.

5. P. G. A. Floris and H. T. Koelink, A commuting q-analogue of the addition formula for disk polynomials, Constr. Approx. 13 (1997), 511-535.

6. G. Gasper, Positivity and the convolution structure for Jacobi series, Ann. of Math. 93 (1971), 112-118.

7. _ Banach algebras for Jacobi series and positivity of a kernel, Ann. of Math. 95 (1972), 261-280.

8. G. Gasper and M. Rahman, Basic Hypergeometric Series, Cambridge University Press, Cambridge, 1990.

9. L. Gegenbauer, Uber einige bestimmte Integrale, Sitz. Math. Natur. Klasse Akad. Wiss. Wien 70 (1875), 433-443.

10. H. T. Koelink, Addition Formulas for q-Special Functions, Fields Institute Communications 14 (1997).

11. J. Math. 47 (1995), 436-448.

12. The addition formula for continuous $q$-Legendre polynomials and associated spherical elements on the SU(2) quantum group related to Askey-Wilson polynomials, SIAM J. Math. Anal. 25 (1994), 197-217.

13. T. H. Koornwinder, The addition formula for little $q$-Legendre polynomials and the SU(2) quantum group, SIAM J. Math. Anal. 22 (1991), 295-301.

14. P. Laplace, Théorie des attractions des sphéroides et de la figure des planètes, Mem. de l'Acad. Royale des Sciences de Paris, Published in 1785, 113-196; reprinted in Euvres de Laplace 10 (1894), 339-419.

15. M. Rahman and A. Verma, Product and addition formulas for the continuous $q$-ultraspherical polynomials, SIAM J. Math. Anal. 17 (1986), 1461-1474.

16. M. Rahman, A simple proof of Koornwinder's addition formula for the little q-Legendre polynomials, Proc. Amer. Math. Soc. 107 (1989), 373-381.

17. D. Stanton, Orthogonal polynomials and Chevally groups. In: Special Functions: Group Theoretic Aspects and Applications, (R. A. Askey, T. H. Koornwinder, and W. Schempp, eds.), Reidel, Dordecht, 44 (1984), pp.867-879.

18. W. Van Assche and T. H. Koornwinder, Asymptotic behavior for Wall polynomials and the addition formula for the little q-Legendre polynomials, SIAM J. Math. Anal. 22 (1991), 302-311. 
19. N. Ja. Vilenkin, Special Functions and the Theory of Group Representations, Translations of Math. Monographs, Vol 22, AMS, Providence, RI, 1968.

20. M. Ja. Vilenkin and A. U. Klimyk, Representations of Lie Groups and Special Functions, 3 volumes, Kluwer, Dordrecht, 1991-1993.

21. E. T. Whittaker and G. N. Watson, A Course of Modern Analysis, Cambridge University Press, Cambridge, 1927.

School of mathematics and Statistics, Carleton University, OtTaWa, Ontario, K1S 5B6, Canada

E-mail: mrahman@math.carleton.ca, qtariq@mathstat.carleton.ca 